\title{
Inteligencia emocional y actitud hacia el proyecto educativo institucional de los docentes de las instituciones educativas del distrito de Huaura
}

\author{
Inteligencia emocional y actitud hacia el proyecto educativo institucional \\ de los docentes de las instituciones educativasdel distrito de Huaura
}

Jesús Retto M. ${ }^{1}$

(RECIBIDO 15-10 2014, ACEPTADO 02-12-2014)

\begin{abstract}
RESUMEN
Se compara la inteligencia emocional de una muestra probabilística de 150 docentes del distrito de Huaura (departamento de Lima), con sus actitudes frente al proyecto educativo institucional de sus centros de trabajo, a fin de detectar si existe algún vínculo entre estas dos variables. Asimismo, se investiga qué tipo de actitudes son las que tienen mayor incidencia. Es un estudio de enfoque cuantitativo, alcance correlacional y diseño no experimental de tipo transeccional. Para la medición de variables se aplicó la escala de inteligencia emocional adaptada de Ruiz, 2004; y la escala de actitudes hacia el proyecto educativo institucional. Los resultados permiten concluir que dichas variables no están asociadas entre sí, y que la actitud predominante es la de aceptación.
\end{abstract}

Palabras clave: inteligencia emocional, actitudes, proyecto educativo institucional.

\begin{abstract}
Emotional Intelligence of a Probabilistic sample of 150 teachers from the Huaura district (Department of Lima) is contrasted with their attitudes toward the Institutional Educational Project of their schools in order to detect if there is a link between these two variables. At the same time, one investigates what kind of attitudes are the ones which have numeric predominance. This is a research with Quantitative approach, Correlacional scope, and Non Experimental design of Transeccional type. To size variables we applied the Emotional Intelligence Scale adapted from Ruiz, 2004; and the Attitudes Scale concerning the Institutional Educational Project. The statistical results let us conclude that such variables are not correlated between them, and that acceptation is the attitude with prevalence.
\end{abstract}

Keywords: Emotional intelligence, attitudes, institutional educational project.

1 Magíster en psicología por la UNMSM. E-mail: graceretto168@hotmail.com 


\section{INTRODUCCIÓN}

Es indudable que el factor decisivo para sacar adelante cualquier proyecto es el factor humano. Son los grupos de trabajo quienes en cualquier entidad tienen en sus manos la posibilidad de hacer realidad desde un pequeño objetivo doméstico hasta un lejano sueño o visión corporativa. Y también de que dichos proyectos se queden solo como buenas ideas escritas en un papel. Ciertamente la preparación académica y la amplitud de conocimientos de los miembros del grupo son elementos importantes; pero a la par hay otro conjunto de elementos menos tomados en cuenta y mucho más personales que pueden empujar al proyecto en definitiva hacia el logro o hacia el fracaso. Elementos como el estado de ánimo, la motivación, el optimismo, las relaciones personales y la tolerancia en un grupo de trabajo juegan un papel crucial en la realización de proyectos (Vivas, 2004).

En las escuelas y colegios peruanos de hoy, es frecuente observar el amplio reconocimiento que los docentes hacen del proyecto educativo institucional, como herramienta básica para gestionar con eficacia sus centros de trabajo. Pero a pesar de este hecho, también se observa que junto con la cotidiana problemática de orden pedagógico y administrativo propia de cada institución educativa, subyace la tarea no resuelta del pobre manejo de discordancias e incompatibilidades en cuanto a sentimientos, creencias, emociones y estados de ánimo de los docentes, hechos que terminan por entorpecer y hasta lapidar sus proyectos (Hidalgo y Cuba, 1999).

Por lo general en las instituciones educativas -especialmente públicas- hay un clima institucional muy tenso, con relaciones interpersonales deterioradas entre los miembros del colectivo docente que hacen muy difícil su comunicación, coordinación y consenso final para lograr acuerdos (Vásquez, 2007). A la luz de tal razonamiento, el principal objetivo de este estudio ha sido establecer si hay alguna relación entre inteligencia emocional y actitud hacia el proyecto educativo institucional por parte de los docentes de las instituciones educativas del distrito de Huaura (departamento de Lima).

Consideramos que los principales hallazgos de esta investigación pueden coadyuvar en el diseño de nuevas políticas de gestión educativa, promoviendo el cultivo de la inteligencia emocional en los docentes a fin de que tengan una participación más activa y productiva en la ejecución de proyectos esenciales para sus centros laborales, como es el caso del proyecto educativo institucional.

\section{MÉTODO}

Enfoque: Cuantitativo

Diseño: No experimental

Tipo de diseño no experimental: Transeccional correlacional; porque se realiza una medición en un determinado y único momento, sin indagar por la causa de la relación entre variables (Hernández, Fernández y Baptista, 2010). 
Alcance de la investigación: Correlacional

Unidad de análisis: Docentes

Población de estudio: Los 245 docentes que laboran en el Cercado del distrito de Huaura (según el padrón oficial de instituciones educativas de la Dirección Regional de Educación Lima - Provincias). Dirección de Gestión Institucional Área de Estadística.

Muestra: Se eligió contar con una muestra probabilística. Para determinar el tamaño de la muestra se utilizó el programa STATS ${ }^{\circledR}$, con las características que se mencionan a continuación:

Universo > 245 docentes que laboran en el Cercado de Huaura.

Error máximo aceptable $>5 \%$

Porcentaje estimado de la muestra $>50 \%$

Nivel deseado de confianza> 95

Tamaño calculado $>149.8332$

Tamaño redondeado de la muestra: 150 docentes

\section{Instrumentos de medición}

Para medir la variable inteligencia emocional, se aplicó la escala de inteligencia emocional (adaptada de la escala MINDS para inteligencias múltiples, de César Ruiz Alva, 2004). Para medir la variable Actitud hacia el Proyecto Educativo institucional, se diseñó y aplicó la escala de actitudes hacia el proyecto educativo institucional. Para esta tarea se realizó la correspondiente prueba piloto, la que fue sometida a los análisis de validez, confiabilidad, y análisis factorial de sus componentes.

\section{RESULTADOS}

Como se puede apreciar en las tablas 1 y 2, de los 150 docentes encuestados, 125 alcanzaron un nivel medio de inteligencia emocional (83.33\%), y de esa misma muestra, 103 docentes revelaron una actitud de aceptación hacia el proyecto educativo institucional (66.66 \%). Estos grupos representan las dos mayorías para cada variable estudiada. A continuación y bastante distantes del primer grupo, figuran 28 docentes quienes muestran una actitud de rechazo hacia el proyecto educativo institucional (18.66\%). Y para el caso de la variable inteligencia emocional, el segundo grupo también se ubica bastante alejado del primero, y lo conforman 15 docentes (10\%), quienes exhiben un nivel bajo de Inteligencia Emocional. Es oportuno mencionar que ambas variables fueron medidas no solo sobre una misma muestra, sino de manera simultánea. Las dos escalas estaban impresas una al anverso y la otra al reverso, en la misma hoja de aplicación. 
Tabla 1. Cantidades y porcentajes obtenidos para la variable inteligencia emocional.

\begin{tabular}{ccc}
\hline NIVEL DE INTELIGENCIA EMOCIONAL & $\mathrm{N}$ & $\%$ \\
\hline BAJO & 15 & 10 \\
MEDIO & 125 & 83.33 \\
ALTO & 10 & 6.66 \\
Total & 150 & 99.99 \\
\hline
\end{tabular}

Tabla 2. Cantidades y porcentajes obtenidos para la variable actitud hacia el proyecto educativo institucional.

\begin{tabular}{ccc}
\hline ACTITUD HACIA EL PEI & $\mathrm{N}$ & $\%$ \\
\hline FUERTE RECHAZO & 3 & 2 \\
RECHAZO & 28 & 18.66 \\
INDIFERENCIA & 7 & 4.66 \\
ACEPTACIÓN & 103 & 68.66 \\
ENTUSIASMO & 9 & 6 \\
Total & 150 & 99.98 \\
\hline
\end{tabular}

Asimismo, se tomó la muestra total $(\mathrm{N}=150)$ para someterla a un análisis estadístico que permitiese detectar la existencia de una posible relación entre las variables inteligencia emocional y actitud hacia el proyecto educativo institucional. Con este propósito se aplicó el coeficiente de correlación de Pearson ( $r$ ), prueba paramétrica que arrojó como resultado el valor 0.04 (véase la tabla 3). Tomando en cuenta el cuadro de interpretaciones propuesto por Hernández, Fernández y Baptista (2010), dicho valor indica que: "no existe correlación alguna entre las variables contrastadas".

Tabla 3. Análisis estadístico de la relación inteligencia emocional - actitud hacia el proyecto educativo institucional, aplicando el coeficiente de correlación de pearson ( $r$ ).

\begin{tabular}{llcc}
\hline & & Actitud & IE \\
\cline { 2 - 4 } Actitud & Correlación de Pearson & 1 &, 044 \\
& Sig. (bilateral) & &, 594 \\
& $\mathrm{~N}$ & 150 & 150 \\
\hline \multirow{2}{*}{ IE } & Correlación de Pearson &, 044 & 1 \\
& Sig. (bilateral) &, 594 & \\
& $\mathrm{~N}$ & 150 & 150 \\
\hline
\end{tabular}


En un ulterior análisis, y tomando en consideración únicamente los puntajes que indicaban una actitud positiva hacia el PEI $(N=112)$, se aplicó nuevamente la correlación de Pearson, y se obtuvo el valor 0.1 (tabla 4) el que debe interpretarse como que "existe una correlación positiva muy débil entre las variables" (Hernández, Fernández y Baptista, 2010). En consecuencia, los resultados de estos dos últimos análisis estadísticos aportan evidencias en contra de la hipótesis general de esta investigación, en la que se plantea tentativamente que existirían relaciones significativas entre la inteligencia emocional de los docentes de las Instituciones educativas del distrito de Huaura y su actitud hacia el proyecto educativo institucional.

Tabla 4. Análisis estadístico de la relación inteligencia emocional - actitud positiva hacia el proyecto educativo institucional, aplicando el coeficiente de correlación de Pearson (r).

\begin{tabular}{cccc}
\hline & & Actitud & IE \\
\cline { 2 - 4 } Actitud & Correlación de Pearson & 1 &, 106 \\
& Sig. (bilateral) & &, 268 \\
& $\mathrm{~N}$ & 112 & 112 \\
\cline { 2 - 4 } IE & Correlación de Pearson &, 106 & 1 \\
& Sig. (bilateral) &, 268 & \\
& $\mathrm{~N}$ & 112 & 112 \\
\hline
\end{tabular}

Observando la figura 1, se aprecia que los valores tienden ligeramente a agruparse hacia la derecha de la curva normal o campana de Gauss, con una asimetría negativa de -.277 . Esto significa que la mayoría de valores se encuentran ubicados por encima de la Media. En cuanto al criterio de Curtosis, se notan variaciones con respecto a una curva normal. Se trata más bien de una curva positiva, cuyo valor es de .072 con un error típico de Curtosis de 394. Su varianza es amplia, alcanzando el valor de 296.453 .

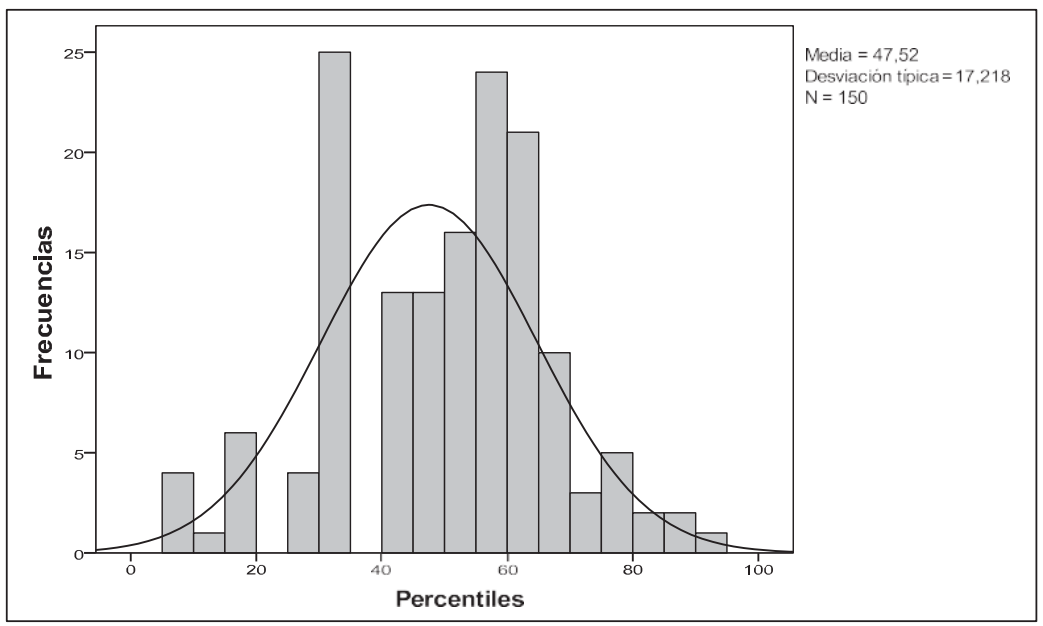

Figura 1. Histograma general para la variable inteligencia emocional. 
Observando la figura 2, se puede notar que hay una ligera agrupación de los valores hacia la derecha de la curva normal, denotando una asimetría negativa de -.523 (Con un error típico de asimetría de .198). Esto significa que la mayoría de valores se encuentran ubicados sobre la media. En cuanto al criterio de Curtosis, se observa que hay una mayor elevación con respecto a una curva normal, lo que nos indica que se trata de una Curtosis positiva, cuyo valor es 1.391 (Con un error típico de Curtosis de .394). La varianza (desviación típica al cuadrado) alcanza el valor de 101.546.

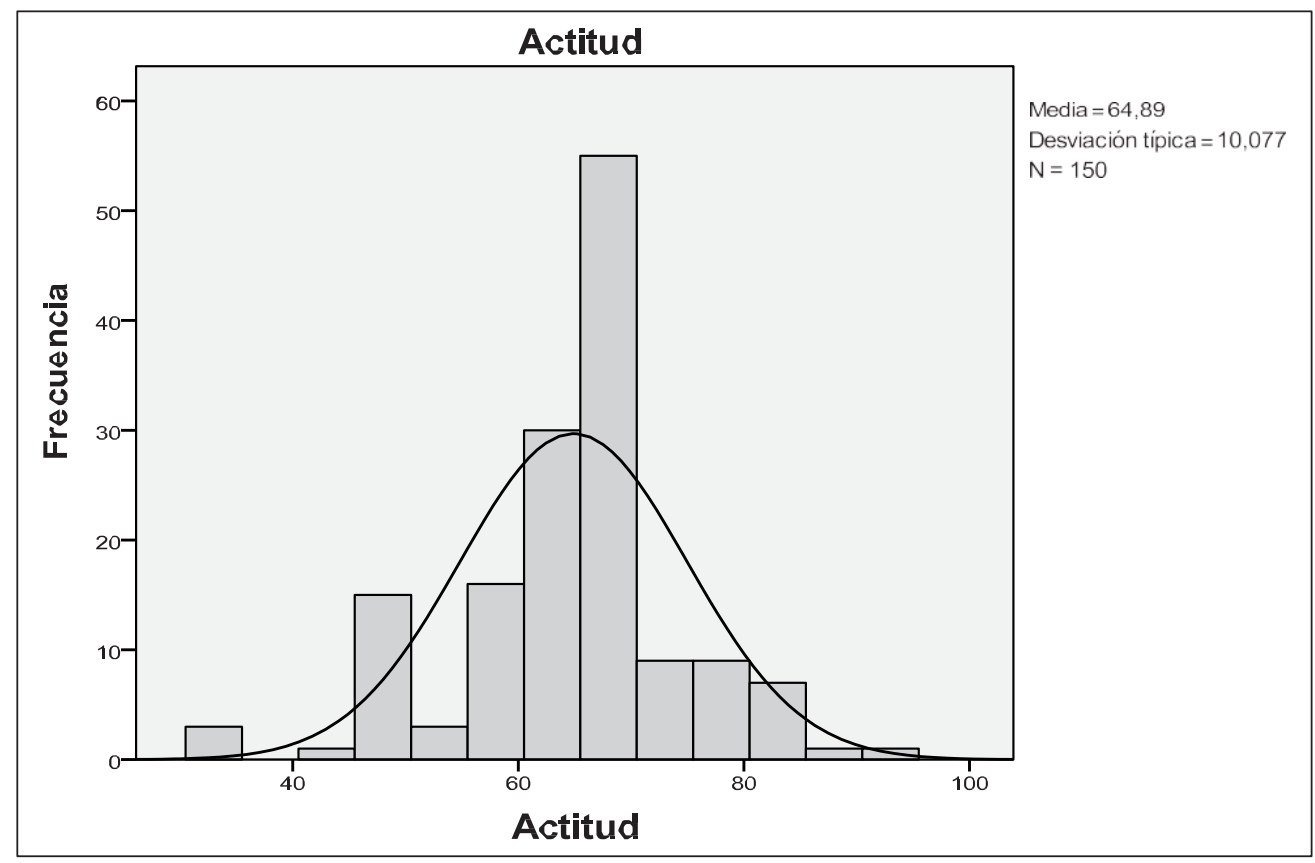

Figura 2. Histograma general para la variable actitud hacia el proyecto educativo institucional.

\section{DISCUSIÓN}

Se ha trabajado con hipótesis de investigación de tipo correlacional para saber cómo es el comportamiento de una variable al conocer el comportamiento de otra. Bajo esa premisa, los resultados obtenidos y los consiguientes análisis realizados han permitido constatar que la variable inteligencia emocional y la variable actitud hacia el proyecto educativo institucional por parte de los docentes de la muestra no guardan correlación alguna, pudiéndose establecer los antecedentes expuestos al inicio de este estudio solo como un factor de casualidad. No hay evidencia científica acerca de la existencia de un factor causal que relacione una variable con la otra. Así lo demuestran, por ejemplo, los resultados obtenidos con el coeficiente de correlación de Pearson ( $r$ ); prueba paramétrica que fue aplicada tanto para el total de la muestra, como solo para aquellos docentes con puntajes que indicaban una actitud positiva hacia el proyecto educativo institucional. 
En la hipótesis general de esta investigación se planteaba tentativamente que podía haber una relación entre la inteligencia emocional de los docentes de las instituciones educativas del distrito de Huaura y su actitud hacia el proyecto educativo institucional, en razón de que un docente con un nivel de inteligencia emocional medio o alto tiene mayor probabilidad de fomentar la existencia de un clima laboral positivo, de más respeto, confianza, lealtad, y de mejor disposición para resolver la problemática laboral (Stys \& Brown, 2004; y Burger, 2009), componentes interpersonales que podrían contribuir a que los profesores tengan en consecuencia una actitud más positiva hacia el diseño y aplicación de instrumentos de gestión educativa fundamentales en sus escuelas y colegios, como es el proyecto educativo institucional. Sin embargo, no fue posible conseguir una prueba científica que respalde y dé veracidad medible a tal razonamiento, quedando lo anterior solo como una especulación. Al respecto, coincidimos con lo planteado por Corral (1998), en que sería provechoso diseñar y aplicar nuevos instrumentos que permitan expandir la medición actitudinal al uso de registros no verbales, de manera que se pueda recoger información a partir de posturas, gestos, manipulaciones espaciales y reacciones fisiológicas, como manifestaciones de actitudes-motivos, además de los clásicos autor reportes verbales; utilizando para ello estudios longitudinales y no solo transversales, de manera que se pueda apreciar la persistencia de una actitud en el tiempo. Sin duda que estos nuevos enfoques afinarían los resultados.

En lo que respecta al planteamiento de que la actitud hacia el proyecto educativo institucional con mayor incidencia entre los docentes de la muestra es la de aceptación, en efecto, se comprobó su validez, puesto que del total de 150 docentes que conformaban la muestra, 103 demostraron tener tal actitud (68.66\%), según la puntuación obtenida con el instrumento respectivo. Más aún, si se unen tales resultados con los de aquellos que demostraron entusiasmo, se llega a 112 docentes, lo que hace un $\mathbf{7 4 . 6 6 \%}$ del total de la muestra. Por otro lado, es posible observar, en este estudio, la primacía del grupo de docentes con una actitud positiva hacia el proyecto educativo institucional (aceptación y entusiasmo $=$ 74.66\%), que destaca nítidamente frente al grupo de docentes con una actitud negativa hacia este proyecto (rechazo y fuerte rechazo $=20.66 \%$ ). Coincidimos con los planteamientos de Extremera y Fernández-Berrocal (2004), en los que se destaca que adecuados niveles de inteligencia emocional ayudan a afrontar con mayor éxito la problemática laboral a la que están sometidos los profesores en el contexto educativo. Es decir, el cultivo de la inteligencia emocional como factor clave para asumir actitudes más positivas en las aulas, o fuera de ellas.

\section{CONCLUSIONES}

1. De acuerdo a los análisis estadísticos realizados en esta investigación, no existe correlación entre las variables inteligencia emocional y actitud hacia el proyecto educativo institucional por parte de los docentes de la muestra estudiada. se rechaza la validez de la hipótesis general. 
2. No obstante la anterior conclusión, algunos hallazgos en esta investigación, y también reportes de investigaciones antecedentes, han observado el hecho de que adecuados niveles de inteligencia emocional en el profesorado ayudan a asumir actitudes más positivas en sus entornos laborales.

3. Son muy pocos los docentes que permanecen indiferentes ante los procesos de gestión institucional de sus escuelas y colegios, pues en su mayoría adoptan una posición definida, una actitud clara, principalmente de aceptación ante propuestas como la construcción del proyecto educativo institucional.

4. Es notable el predominio de docentes con niveles medios de inteligencia emocional, sobre los niveles extremos, ya sean estos altos o bajos.

5. Los docentes reconocen la importancia del proyecto educativo institucional como instrumento básico de gestión tanto pedagógica como institucional de sus centros laborales, aunque se trate de una tarea complicada.

\section{REFERENCIAS BIBLIOGRÁFICAS}

Alcócer, S. (2002). Recursos Humanos, Organización e Inteligencia Emocional. Revista Peruana de Psicología, 7(12), 72-80.

American Psychological Association (2010). Manual de Publicaciones de la American Psychological Association. (3aㅡ. Ed.) Washington DC. USA: Manual Moderno.

Anadón, Ó. (2006). Inteligencia emocional percibida y optimismo disposicional en estudiantes universitarios. Revista Electrónica Interuniversitaria de Formación del Profesorado, 9(1), 1-13. Recuperado de http://www.aufop.com/aufop/home

Araujo, M. (2007). Inteligencia Emocional y Desempeño Laboral en las instituciones de Educación Superior Públicas. (Tesis de doctorado, Universidad Rafael Belloso Chacín) Recuperado de http://www.urbe.edu/portal-biblioteca/basesdedatos-urbe/tesis/

Barent, J. (2005). Principals' levels of Emotional Intelligence as an influence on school culture. (Tesis de doctorado, Montana State University). Recuperado de http://etd. lib.montana.edu/etd/view/item/206

Bar-On, R. (2006). The Bar-On Model of Emotional-Social Intelligence (ESI). Psicothema 18(Supl.), 13-25. Recuperado de www.eiconsortium.org

Bedwell, S. (2003, abril). Emotional Intelligence: Personality revisited or something else? Researching emotional intelligence in a personnel psychology context. Simposio realizado en el Annual meeting of the Society of Industrial and Organizational Psychology, Orlando, FL., USA.

Birknerová, Z. (2011). Social and Emotional Intelligence in School Environment. Asian Social Science 7(10), 241-248. Recuperado de http://www.ccsenet.org/journal/ index.php/ass

Briñol, P., Horcajo, J., Becerra, A., Falces, C. y Sierra, B. (2002). Cambio de actitudes implícitas. Psicothema 14(4), 771 - 775. Recuperado de http://www.psicothema. com/psicothema.asp?id=3202 
Buitrón, S. y Navarrete, P. (2008). El docente en el desarrollo de la inteligencia emocional: reflexiones y estrategias. Docencia Universitaria - Universidad Peruana de Ciencias Aplicadas, 4(1), 1-8. Recuperado de http://www.upc.edu.pe/docencia-universitaria/

Burger, T. (2009). Emotional Intelligence and well-being in teachers. (Tesis de maestría, Stellenbosch University). Recuperado de http://library.sun.ac.za/index.htm

Cabanillas, W. (2002). Modelos en Inteligencia Emocional: Más allá del legado de Goleman. Revista Peruana de Psicología, 7(12), 35-41.

Cabello, Rosario; Ruiz-Aranda, Desirée \& Fernández-Berrocal, Pablo (2010). Docentes emocionalmente inteligentes. REIFOP, 13 (1). Recuperado de http://www.aufop.com

Carmeli, A. (2003). The relationship between emotional intelligence and work attitudes, behavior and outcomes. Journal of Managerial Psychology 18(8), 78-813. Recuperado de http://www.emeraldinsight.com/0268-3946.htm

Cépeda, N. (2009). Diversidad cultural de los maestros peruanos: un potencial para una educación intercultural. (Tesis de maestría, Pontificia Universidad Católica del Perú). Recuperado de http://tesis.pucp.edu.pe/repositorio/bitstream/handle/123456789/1164

Cerny, C, \& Kaiser, H. (1977). A study of a measure of sampling adequacy for factoranalytic correlation matrices. Multivariate Behavioral Research, 12(1), 43-47.

Recuperado de https://www-304.ibm.com/support/docview.wss?uid=swg21479963

Chávez, H. (2006). Proyecto Educativo Institucional (Informe del Ministerio de Educación del Perú). Lima, Perú: MINEDU.

Christie, A., Jordan, P., Troth, A., \& Lawrence, S. (2007). Testing the links between emotional intelligence and motivation. Journal of Management \& Organization, 13(3), 212 - 226. Recuperado de http://jmo.e-contentmanagement.com/

Consejo Nacional de Educación (2006). Proyecto Educativo Nacional al 2021 (Informe gubernamental). Lima, Perú: CONADE.

Consejo Nacional de Educación (2011). Proyecto Educativo Nacional 2007-2010 Balance y Recomendaciones (Informe gubernamental). Lima, Perú: CONADE.

Cook, C. (2006). Effects of Emotional Intelligence on Principals' Leadership performance. (Tesis de doctorado, Montana State University). Recuperado de http://etd.lib.montana. edu/etd/view/item/79

Cornejo, R. (2009). Condiciones de trabajo y bienestar / malestar docente en profesores de Enseñanza Media de Santiago de Chile. Educaçâo e Sociedade, 30(107), 409 - 426. Recuperado de http://www.cedes.unicamp.br

Coronel, E. y Ñaupari, F. (2011). Relación entre inteligencia emocional y desempeño docente en aula en la Universidad Continental de Huancayo. Apuntes en Ciencias Sociales, 01(01), 27-32. Recuperado de http://www.ucci.edu.pe/portal/pdfs/revista_ apuntes/apuntes_v1n1_coronel_naupari.pdf

Corral, V. (1998). Un análisis crítico del concepto "Actitudes". Propuesta de un enfoque naturalista. Revista Mexicana de Análisis de la Conducta, 24(1), 1-16. Recuperado de http://www.revistas.unam.mx/index.php/rmac 
Craig, J. (2008). The relationship between the Emotional Intelligence of the principal and teacher job satisfaction. (Tesis de doctorado, University of Pennsylvania). Recuperado de http://www.library.upenn.edu/

Cueto, S., Andrade, F. y León, J. (2003). Las actitudes de los estudiantes peruanos hacia la lectura, la escritura, la matemática y las lenguas indígenas. (Informe $\mathrm{N}^{\circ} 44$ ) Lima, Perú: GRADE.

Culver, D. (1998, Noviembre). A Review of Emotional Intelligence by Daniel Goleman: Implications for Technical Education. Frontiers in Education Conference. Simposio realizado en FIE '98. 28th Annual, New York, USA.

Elejabarrieta, F. e Iñiguez, L. (1984). Construcción de Escalas de Actitud tipo Thurstone y tipo Likert. Recuperado de http://www.4shared.com/document/S-e6_SGp/ Elejabarrieta_F1J_e_Iguez_L_C.html

Espinosa, J. y Román, T. (1998). La medida de las actitudes usando las técnicas de Likert y de Diferencial Semántico. Enseñanza de las Ciencias, 16(3), 477- 484. Recuperado de http://ensciencias.uab.es/

Evers, W., Brouwers, A. \& Tomic, W. (2002). Burnout and self-efficacy: A study on teachers' beliefs when implementing an innovative educational system in the Netherlands. British Journal of Educational Psychology 72, 227-243. Recuperado de www.bps.org.uk

Extremera, N. y Fernández-Berrocal, P. (2004). La importancia de desarrollar la inteligencia emocional en el profesorado. Revista Iberoamericana de Educación, 33(8), 1-9. Recuperado de http://www.rieoei.org/index.php

Fernández, A. (1999, marzo). Estudio de las actitudes del profesorado de los Centros Ordinarios de Vizcaya hacia la integración escolar. Documento presentado en el Simposio Retos en la respuesta al retraso mental en la vida adulta: Formación, oportunidades y calidad de vida. Universidad de Deusto, Bilbao, España.

Foro Educativo (2013). Maestros, Sociedad y Estado. Bases para un Acuerdo Nacional por el Maestro Peruano.(Conferencia Nacional) Lima, Perú: Foro Educativo.

Gabel, R. (2005). Inteligencia Emocional: perspectivas y aplicaciones ocupacionales. (Documento de trabajo $N^{\circ} 16$ de la Universidad ESAN). Lima, Perú: ESAN Ediciones.

García, M. y Llorens, S. (2003, mayo). ¿̇nfluyen los obstáculos laborales en el malestar docente? Octava Jornada de Fomento a la Investigación en Ciencias Humanas y Sociales. Universitat Jaume I, Castellón, España.

Gardner, H. (2003, abril). Multiple Intelligences After Twenty Years. Documento presentado a la American Educational Research Association, Chicago, USA.

Goleman, D. (1998). La práctica de la Inteligencia Emocional. Barcelona, España: Kairós.

Hernández, R., Fernández, C. y Baptista, P. (2010). Metodología de la investigación (5a Ed.). México, D.F., México: McGraw-Hill.

Herrera, J. (2002). Inteligencia Emocional en el trabajo. Revista Peruana de Psicología, 7(12), 63-71.

Hidalgo, L. y Cuba, S. (1999). Construyendo la Nueva Escuela. Proyecto Educativo Instituciona. Volumen I. Lima, Perú: Tarea. 
Hidalgo, L. y Cuba, S. (1999). Construyendo la Nueva Escuela. Proyecto Educativo Instituciona. Volumen II. Lima, Perú: Tarea.

Hasapoglu, H. (20l0). Relación entre las actitudes hacia el cambio de los profesores de aula, sus percepciones de cambio del curriculum constructivista, la implementación de la enseñanza constructivista, y las actividades de aprendizaje en clase, en el nivel escolar primario. (Tesis de maestría, Middle East Technical University) Recuperado de http://www.metu.edu.tr/

Macotela, S., Flores, R. y Seda, I. (2001). Las creencias de docentes mexicanos sobre el papel de la escuela y del maestro. Revista Iberoamericana de Educación, (s.n.) 1-24. Recuperado de http://www.rieoei.org/profesion16.htm

Masías, Y. (2006). Relación entre las actitudes hacia la sexualidad y nivel de conocimiento sobre factores de riesgo de discapacidad prenatal en adolescentes del Cono Sur de Lima. (Tesis inédita de maestría). Universidad Peruana Cayetano Heredia, Lima, Perú.

Ministerio de Educación del Perú (2008). Manual del Director (Vol. 1) Lima, Perú: MINEDU.

Naseer, Z.; Chishti, S.; Rahman, F.; \& Jumani, N. (2011). Impacto de la Inteligencia Emocional en equipos de trabajo de 15 Institutos de Educación Superior de Pakistán. International Online Journal of Educational Sciences, 3(1), 30-46. Recuperado de: http://www.iojes.net/article/magazinearticle.aspx?ID=42

Obando, G. (2008). Participación docente en la toma de decisiones. Una mirada micropolítica. (Tesis inédita de maestría). Pontificia Universidad Católica del Perú, Lima, Perú.

Padilla, K. y Van Driel, J. (2009, septiembre). Relaciones entre los aspectos cognitivos y emocionales de la enseñanza. VIII Congreso Internacional sobre Investigación en la Didáctica de las Ciencias, Revista Enseñanza de las Ciencias, Barcelona, España.

Palomera, R., Fernández-Berrocal, P., y Brackett, M. (2008). La inteligencia emocional como una competencia básica en la formación inicial de los docentes: algunas evidencias. Revista Electrónica de Investigación Psicoeducativa, 6(2), 437-454. Recuperado de http://redie.uabc.mx/vol12no2/contenido-contenido.html

Ponce, C., Bulnes, M., Aliaga, J., Atalaya, M. y Huertas, R. (2005). El síndrome del "quemado" por estrés laboral asistencial en grupos de docentes universitarios. Revista de Investigación en Psicología - UNMSM, 8(2), 87-112.

Prieto, L. y Pirela, L. (2005). La inteligencia emocional en el docente-investigador de LUZ. Encuentro Educacional, 12(3), 395-414. Recuperado de http://www.revistas. luz.edu.ve/index.php/ed

Quintana, A. (2008) Planteamiento del Problema de Investigación: errores de la lectura superficial de libros de texto de metodología. Revista de Investigación en Psicología - UNMSM, 11(1), 239-253.

Reed, T. (2005). Elementary Principal Emotional Intelligence, Leadership Behavior, and Openess: An Exploratory Study. (Tesis de doctorado, Ohio State University), Recuperado de http://etd.ohiolink.edu/view.cgi?acc_num=osu1133194372 
Rego, A. y Fernandes, C. (2005). Inteligencia Emocional: Desarrollo y Validación de un Instrumento de Medida. Revista Interamericana de Psicología, 39(1), 23-38. Recuperado de http://www.psicorip.org/

Retto, G. (2006). El Proyecto Educativo Institucional - PEI. Lima, Perú: Autor.

Reyes, L. (2007). La Teoría de la Acción Razonada: Implicaciones para el estudio de las Actitudes. Investigación Educativa, 2(7), 66-77. Recuperado de http://www.upd. edu.mx/librospub/revistas/invedu07.pdf

Ríos, R. (2004). El trabajo en equipo en las decisiones organizativas. Un estudio de casos en el Centro Educativo Estatal 0019 "San Martín de Porres Velásquez". (Tesis inédita de maestría). Pontificia Universidad Católica del Perú, Lima, Perú.

Robles, E. (2005). Los docentes en el proceso de gestión de un Currículo por Competencias. (Tesis de maestría, Pontificia Universidad Católica del Perú) Recuperado de http:// tesis.pucp.edu.pe/repositorio/handle/123456789/123

Rodríguez, I. (2005). Programa de adiestramiento en Inteligencia Emocional para docentes del Centro de Estudios Avanzados. (Tesis de doctorado, Tecana American University). Recuperado de http://www.tauniversity.org/index.htm

Ruiz, C. (2004). Escala MINDS de Inteligencias Múltiples. Trujillo, Perú: Autor.

Ruiz, J. (2004). El clima laboral y la inteligencia emocional. (Tesis de maestría, Universidad Nacional de Educación “Enrique Guzmán y Valle") Recuperado de http://www.ucci. edu.pe/portal/images/pdfs/revista_apuntes/apuntes_

Salvador, Adelina (2010). Relación de la Inteligencia Emocional en el liderazgo del equipo directivo en las instituciones educativas de la zona de Canto Grande, UGEL № 05, del distrito de San Juan de Lurigancho. (Tesis de maestría, Universidad Nacional Mayor de San Marcos, Lima - Perú) Recuperado de http://www.cybertesis.edu.pe/ sisbib/2010/salvador_aa/pdf/salvador_aa.pdf

Salovey, P. y Mayer, J. (1990). Emotional Intelligence. New Haven, United States: Baywood Publishing Co. Inc.

Serna, C. y Toloza, H. (2009). Actitudes de los maestros frente a la política pública de campos del pensamiento, en la Institución Educativa Tomás Cipriano de Mosquera -Colegio de Excelencia del Distrito Capital-. (Tesis de maestría, Pontificia Universidad Javeriana de Bogotá). Recuperado de http://repository.javeriana.edu. co/handle/10554/422

Siu, E. (2004) El diseño metodológico en la gestión de un Proyecto Educativo Innovador. (Tesis de maestría, Pontificia Universidad Católica del Perú) Recuperado de http:// tesis.pucp.edu.pe/repositorio/handle/123456789/607

Solf, A. (2002). Las emociones en el trabajo. Revista Peruana de Psicología, 7(12), 49-53.

Stys, Y. \& Brown S. (2004). A Review of the Emotional Intelligence Literature and Implications for Corrections. (Informe de Investigación № 150 de la Research Branch Correctional Service of Canada). Recuperado de http://www.cscscc.gc.ca/text/rsrch/ reports/r150/r150_e.shtml 
Tecsi R. (2006). Relación entre la inteligencia emocional y la calidad de las relaciones humanas. (Tesis de maestría, Universidad Nacional de Educación “Enrique Guzmán y Valle") Recuperado de http://www.ucci.edu.pe/pdfs/

Traver, J. y García, R. (2007). Construcción de un cuestionario-escala sobre actitud del profesorado frente a la innovación educativa mediante técnicas de trabajo cooperativo (CAPIC). Revista Electrónica de Investigación Educativa, 9(1), 1-14. Recuperado de http://redie.uabc.mx

Ugarriza, N. (2003). La evaluación de la Inteligencia Emocional a través del Inventario de Bar-On (I-CE) en una muestra de Lima metropolitana. Lima, Perú: Autor.

Vásquez, F. (2007). Inteligencia Emocional en las Organizaciones Educativas. Psicogente, 10(17), 42-59. Recuperado de www.unisimonbolivar.edu.co

Villanueva, J. (2008). La inteligencia emocional rasgo, la autoeficacia para el liderazgo y su vinculación a procesos afectivos grupales, cognitivos y de desempeño.(Tesis de doctorado, Universidad de Salamanca). Recuperado de http://www.usal.es/webusal/

Vivas, M. (2004, mayo). Las Competencias Socioemocionales del Docente: Una mirada desde los formadores de formadores. Competencias profesionales de las titulaciones de Educación. Simposio realizado en la Primera Jornada Universitaria UNED, Madrid, España.

Yarlequé, L. (2004). Actitudes hacia la conservación ambiental en estudiantes de educación secundaria. (Tesis inédita de doctorado). Universidad Nacional Mayor de San Marcos, Lima, Perú. 
Anexo 1

ESCALA DE INTELIGENCIA EMOCIONAL

(Adaptada de la escala MINDS para inteligencias múltiples, de César Ruiz Alva, 2004).

\section{ENCUESTAANÓNIMA}

Sexo:

Edad:

Tiempo de servicios:

Estimado(a) profesor(a), por favor marque UNO de los números que está al lado de cada afirmación, según su parecer.

\section{SIGNIFICADO DE LOSNÚMEROS}

\begin{tabular}{cc}
\hline 0 & No se parece en absoluto a mi. \\
1 & Se parece un poco a mi \\
2 & Se parece bastante a mi \\
3 & Se parece totalmente a mi \\
\hline
\end{tabular}

1 Siento que agrado y caigo bien a personas de todas las edades.

2 Las debilidades que observo en los demás, también las busco en mí.

3 Respondo a los demás con entusiasmo, sin prejuicios o medias palabras.

4 Con frecuencia pienso en la influencia que tengo sobre los demás.

5 Me encanta compartir experiencias con una variedad de personas. Creo firmemente que soy responsable de quién soy: un producto de mis elecciones personales.

7 Me siento cómodo(a) disfrutando de situaciones sociales nuevas. La vida hay que aprovecharla al máximo, y por eso no malgasto mi tiempo en cosas sin importancia.

9 Siento que caigo bien a los niños desde el primer momento que me ven. Me encanta leer sobre los grandes filósofos que han escrito sobre sus inquietudes, luchas y alegría de vivir.

11 Puedo reconocer sin problemas los méritos y éxitos de los demás.

12 Con frecuencia pienso en la condición humana y en el lugar que yo ocupo en este mundo.

13 Me doy cuenta rápidamente cuando alguien quiere manipular a otro.

17 Pienso que todos somos iguales, y no desmerezco a nadie.

18 Creo que soy una persona completamente honesta conmigo mismo(a). 
Anexo 2

ESCALA DE ACTITUDES HACIA EL PROYECTO EDUCATIVO INSTITUCIONAL (P.E.I.)

Sexo:

Edad:

Tiempo de servicios:

Estimado(a) profesor(a), marque solo UNA de las letras que están al lado de cada afirmación según su parecer.

\section{SIGNIFICADO DE LAS LETRAS}

TD: Estoy totalmente en desacuerdo.

D: Estoy en desacuerdo.

E: Tengo mis dudas / No lo sé.

A: Estoy de acuerdo.

TA: Estoy totalmente de acuerdo.

\begin{tabular}{|c|c|c|c|c|c|c|}
\hline 1 & El PEI es un documento importante. & TD & $\mathrm{D}$ & E & A & TA \\
\hline 2 & Las reuniones para trabajar el PEI son interesantes. & TD & D & $\mathrm{E}$ & A & TA \\
\hline 3 & $\begin{array}{l}\text { El PEI puede dar muchas oportunidades de mejoramiento a las escuelas y } \\
\text { colegios. }\end{array}$ & TD & $\mathrm{D}$ & $\mathrm{E}$ & A & TA \\
\hline 4 & Es pesado tener que aportar ideas para llevar adelante proyectos como ese. & TD & $\mathrm{D}$ & E & A & TA \\
\hline 5 & Las reuniones de coordinación son muy largas y aburridas. & TD & D & $\mathrm{E}$ & A & TA \\
\hline 6 & Generalmente son dos o tres los que siempre hablan en las reuniones del PEI. & TD & D & $\mathrm{E}$ & A & TA \\
\hline 7 & Siento que hay mucho que aportar y aprender en esas reuniones. & TD & $\mathrm{D}$ & $\mathrm{E}$ & A & TA \\
\hline 8 & Casi nunca toman en cuenta las ideas de todos. & TD & $\mathrm{D}$ & $\mathrm{E}$ & A & TA \\
\hline 9 & $\begin{array}{l}\text { Creo que lo del PEl le compete más que nada a los directores, no a los } \\
\text { profesores de aula. }\end{array}$ & TD & $\mathrm{D}$ & E & A & TA \\
\hline 10 & Esas reuniones con frecuencia terminan en discusiones. & TD & D & $\mathrm{E}$ & A & TA \\
\hline 11 & EI PEI puede mejorar la calidad del servicio educativo. & TD & D & $\mathrm{E}$ & A & TA \\
\hline 12 & Preferiría no comprometerme en asumir cargos que tengan que ver con el PEI. & TD & $\mathrm{D}$ & $\mathrm{E}$ & A & TA \\
\hline 13 & Todos queremos hacer realidad lo planteado en el PEI. & TD & $\mathrm{D}$ & $\mathrm{E}$ & A & TA \\
\hline 14 & $\begin{array}{l}\text { Sospecho que muy pocos colegas realmente tienen interés en diseñar o } \\
\text { ejecutar el PEI. }\end{array}$ & TD & D & $\mathrm{E}$ & A & TA \\
\hline 15 & Hay entusiasmo en ejecutar lo planteado en el PEI. & TD & $\mathrm{D}$ & $\mathrm{E}$ & A & TA \\
\hline 16 & Trabajando el PEI es posible conocer mejor nuestra realidad educativa. & TD & D & $\mathrm{E}$ & A & TA \\
\hline 17 & $\begin{array}{l}\text { Por gusto se pierde tiempo en hacer el PEI, cuando al final nunca se lo } \\
\text { lleva a la práctica. }\end{array}$ & TD & D & E & A & TA \\
\hline 18 & En las reuniones de trabajo hay magníficas ideas y aportes de los colegas. & TD & $\mathrm{D}$ & $\mathrm{E}$ & A & TA \\
\hline 19 & Creo que en la institución hay asuntos más urgentes que el PEI. & TD & D & $\mathrm{E}$ & A & TA \\
\hline 20 & $\begin{array}{l}\text { Pienso que a pesar de las dificultades, el PEI terminará siendo una feliz } \\
\text { realidad. }\end{array}$ & TD & D & $\mathrm{E}$ & A & TA \\
\hline
\end{tabular}

